\title{
La vitalité des spermatozoïdes
}

\author{
T. FORGES, P. MONNIER-BARBARINO, B. FOLIGUET
}

Centre d'Assistance Médicale à la Procréation, Maternité Régionale, NANCY

\section{RÉSUMÉ}

Malgré l'existence de nombreux moyens d'étude de la vitalité des spermatozoïdes, la plupart restent dans le domaine de la recherche biomédicale. Ce sont surtout les tests classiques, tels que le test à l'éosinenigrosine et le test de gonflement flagellaire en milieu hypo-osmolaire (HOST) qui permettent d'apprécier le pourcentage de vitalité des spermatozoïdes en spermiologie courante. Un résultat anormalement bas définit la nécrozoospermie, dont l'interprétation est souvent difficile, mais permet d'orienter le bilan masculin vers la recherche de certains contextes étiologiques, notamment celui des infections génitales chroniques.

L'étude de la vitalité des spermatozoïdes constitue également l'élément-clé dans le choix des spermatozoïdes susceptibles d'être utilisés en vue d'une micro-injection intracytoplasmique. Dans cette indication, seuls les tests en milieu hypo-osmolaire sont applicables. Une analyse individuelle de chaque spermatozoïde avec un temps de séjour minimal dans la solution hypo-osmolaire semble actuellement préférable.

L'exploration de la vitalité des spermatozoïdes concerne désormais également l'apoptose. Cette forme de mort cellulaire peut être mise en évidence dans les spermatozoïdes par différents moyens techniques et pose le problème de la sélection de spermatozoïdes utilisables en assistance médicale à la procréation selon des critères d'intégrité non seulement membranaire, mais aussi génomique.

Mots clés : Vitalité, Spermatozoïde; éosine, HOST, ICSI, Apoptose

\section{INTÉRÊTS DES TESTS DE VITALITÉ DES SPERMATOZOÏDES}

La mise en œuvre de tests biologiques destinés à apprécier la vitalité des spermatozoïdes humains répond à plusieurs objectifs. En spermiologie courante, différentes techniques, basées essentiellement sur la perméabilité ou le pouvoir osmorégulateur de la membrane plasmique, peuvent être utilisées pour établir le pourcentage de vitalité d'une population de spermatozoïdes. Une valeur anormalement basse de ce dernier définit le diagnostic d'une nécrozoospermie, dont l'interprétation s'avère souvent difficile.

Un deuxième champ d'application de ces explorations concerne la fécondation in vitro assistée par micro-injection intracytoplasmique de spermatozoïdes (ICSI), où certains de ces tests peuvent aider le biologiste dans le choix du spermatozoïde à micro-injecter, lorsque tous les spermatozoïdes sont immobiles.

Enfin, un intérêt croissant est actuellement porté à la mise en évidence des processus apoptotiques dans les spermatozoïdes dans le but de pouvoir sélectionner des gamètes à génome intact, en vue de leur utilisation en assistance médicale à la procréation.

Ces trois approches de la vitalité des spermatozoïdes vont être passés en revue.

Correspondance : T. Forges Centre d'Assistance Médicale à la Procréation, Maternité Régionale, 54042 NANCY Cedex

Communication aux 3èmes Journées Nationales Assurance de Qualité en Biologie de Reproduction, $26 \&$ 27 Octobre 2000, Nancy. 


\section{DIAGNOSTIC D'UNE NÉCROZOOSPERMIE}

Le diagnostic d'une nécrozoospermie repose sur la distinction entre spermatozoïdes vivants et non vivants grâce à l'utilisation de colorants vitaux, dont le plus commun est l'éosine, ou de tests de réactivité membranaire en milieu hypo-osmolaire, représentés essentiellement par le HOST (hypo-osmotic swelling test). Les nombreux autres tests disponibles trouvent leurs applications surtout en recherche biomédicale.

L'interprétation d'une nécrozoospermie pose souvent un problème étiologique qui incitera le médecin à orienter la suite du bilan masculin dans plusieurs directions.

\section{TESTS A L'EOSINE}

\section{a) Mécanisme d'action}

Le mécanisme d'action d'un colorant vital est basé sur le fait que la double couche phospholipidique d'une membrane plasmique intacte est imperméable aux molécules du colorant. En revanche, ces dernières peuvent traverser les membranes altérées, s'accumuler dans le cytoplasme ou dans le noyau et colorer ainsi le compartiment cellulaire en question. La vitalité de la cellule est donc attestée par l'exclusion du colorant.

\section{b) Réalisation}

En spermiologie courante, les tests les plus utilisés sont le test à l'éosine seule et le test à l'éosine-nigrosine, introduits par BLOM en 1950. [5]

Pour le test à l'éosine seule, une goutte d'éosine (diluée à $0,5 \%$ dans du sérum physiologique) est mélangée sur une lame avec une goutte de sperme. Après 30 secondes d'incubation, le mélange est soit observé immédiatement entre lame et lamelle, soit étalé par un frottis qui est laissé sécher à l'air pour être observé ensuite au microscope en immersion. [30]

Pour le test à l'éosine-nigrosine, une goutte de sperme est incubée avec l'éosine comme précédemment, puis deux gouttes de nigrosine (diluée à $10 \%$ dans du sérum physiologique) sont ajoutées et après une nouvelle incubation de 30 secondes, un frottis est étalé, séché à l'air et observé au microscope en immersion. L'adjonction de nigrosine permet de contrecolorer le fond de la lame ce qui facilite la lecture au microscope. [30]

Les spermatozoïdes vivants apparaissent incolores sur un fond brun foncé, tandis que les spermatozoïdes non vivants sont colorés en rose par l'éosine au niveau de leur tête, avec une intensité maximale à leur base. Certains spermatozoïdes présentent une légère coloration limitée à leur base, ce qui correspond probablement à un début d'altération de l'intégrité membranaire et donc de leur vitalité (spermatozoïdes "moribonds").

L'Organisation Mondiale de la Santé (OMS) recommande une lecture sur 200 spermatozoïdes et fixe actuellement le seuil de normalité à $50 \%$ de formes vivantes. Le résultat doit être confronté à la mobilité du même échantillon, puisque, théoriquement, le nombre de spermatozoïdes non vivants ne peut être supérieur au nombre d'immobiles.

\section{LE HOST (HYPO-OSMOTIC SWELLING TEST)}

\section{a) Mécanisme d'action}

Ce test est basé sur le caractère semi-perméable d'une membrane cellulaire intacte. En présence d'un milieu extracellulaire hypoosmolaire, il se produit un influx d'eau dans la cellule, de manière à rééquilibrer la pression osmotique de part et d'autre de la membrane. Ce phénomène se traduit par un gonflement de la cellule visible en microscopie optique. Une cellule dont la membrane est altérée, perd cette capacité osmorégulatrice et, par conséquent, ne gonfle pas en milieu hypo-osmolaire.

\section{b) Réalisation}

Le HOST a été initialement proposé comme un test fonctionnel des spermatozoïdes, puis comme test de vitalité par Jeyendran en 1984 [14]. Selon les recommandations de l'OMS, la solution hypo-osmolaire contient $7,35 \mathrm{~g}$ de citrate de sodium et $13,51 \mathrm{~g}$ de fructose dans un litre d'eau distillée. Elle peut être aliquotée et stockée à $-20^{\circ} \mathrm{C}[30]$.

Pour le test, un échantillon de $0,1 \mathrm{ml}$ de sper- 
me est mélangé avec $1 \mathrm{ml}$ de solution hypoosmolaire et incubé à $37^{\circ} \mathrm{C}$ pendant au moins 30 minutes (120 minutes au maximum). Une goutte de cette préparation est ensuite observée sous un microscope équipé d'un contraste de phase.

Les spermatozoïdes vivants présentent un influx d'eau au niveau de leur flagelle, entraînant une distension membranaire et un recourbement des fibres denses. Ce phénomène se traduit, à des degrés variables, par un gonflement et une incurvation du flagelle. Schématiquement, ces modifications peuvent prendre 6 aspects différents (Figure 1). Comme pour le test à l'éosine, l'OMS recommande une lecture sur 200 spermatozoïdes, mais fixe le seuil de normalité à $60 \%$ de formes vivantes pour ce test.
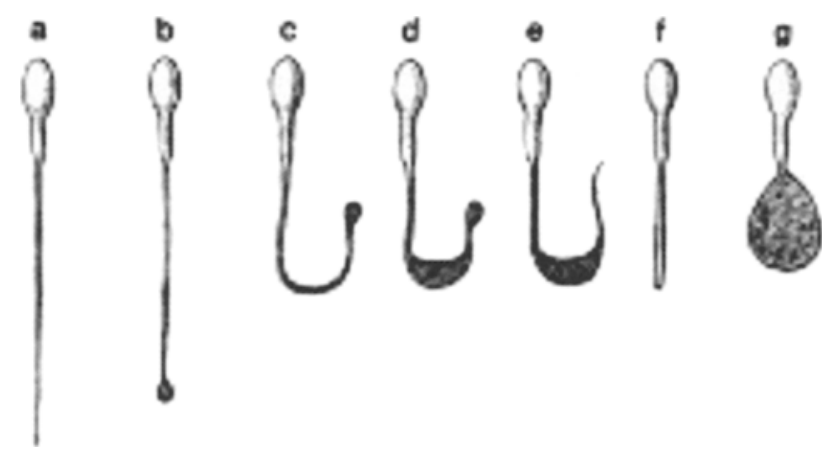

Figure 1: HOST (hypo-osmotic swelling test): types de réaction membranaire du flagelle au stimulus hypo-osmolaire [30].

\section{AUTRES MOYENS D'ÉTUDE DE LA VITALITÉ}

\section{a) Colorants vitaux}

D'autres colorants vitaux sont à la disposition du biologiste pour apprécier la vitalité des spermatozoïdes. Ils sont en général spécifiques du compartiment nucléaire, en se fixant sur l'ADN. Leur détection se fait par fluorescence et nécessite par conséquent des manipulations plus longues et un équipement adéquat (microscope à fluorescence ou cytomètre en flux), ce qui fait qu'ils sont exceptionnellement utilisés en spermiologie courante, mais surtout en recherche.

Parmi ces molécules, il convient de citer essen- tiellement le iodure de propidium qui émet une fluorescence rouge dans le noyau des cellules non vivantes et les colorants Hoechst 33258 et 33342 qui donnent une fluorescence bleue, mais nécessitent une excitation en lumière ultraviolette.

\section{b) Colorants non vitaux}

Certains colorants, comme le SYBR-14, passent également à travers les membranes intactes, mais, pour une raison encore inconnue, donnent une fluorescence plus intense dans les noyaux des cellules vivantes que dans ceux des cellules altérées [12]

\section{c) Tests enzymatiques}

La vitalité peut également être appréciée en explorant les capacités fonctionnelles du compartiment cytoplasmique. Le diacétate de carboxyfluorescéine (CFDA) ou de carboxy-diméthylfluorescéine (CDMFDA) pénètre dans la cellule où, sous l'action d'une estérase cytoplasmique, le fluorochrome est libéré et devient détectable (fluorescence verte). Dans les cellules altérées, la fluorescence est diminuée parallèlement à la perte des capacités fonctionnelles du cytoplasme [11, 26].

Ces techniques nécessitent des lavages préalables afin d'éliminer le bruit de fond causé par la présence d'estérases dans le liquide séminal, ainsi qu'une lecture à un temps précis, car la quantité de fluorescence varie avec la cinétique de la réaction enzymatique.

Les marqueurs enzymatiques à la fluorescéine comme les colorants non vitaux sont le plus souvent associés à un colorant vital de type Hœchst ou iodure de propidium [3, 12]. Dans les spermatozoïdes en voie d'altération, ce double marquage permet d'observer un changement de fluorescence du vert au rouge, en passant par une fluorescence orangée intermédiaire.

\section{d) Microscopie éléctronique}

L'étude ultrastructurale des spermatozoïdes en microscopie électronique permet de confirmer le diagnostic de nécrozoospermie en montrant des altérations caractéristiques de la dégénérescence cellulaire qui touchent tous les organites cellulaires des spermatozoïdes [31]: 
- Fragmentation, voire disparition complète de la membrane plasmique ;

- Raréfaction de la matrice de l'acrosome et vésiculation de la membrane acrosomiale ;

- Caryolyse ;

- Gonflement des mitochondries et fragmentation de leurs crêtes ;

- Raréfaction des colonnes longitudinales et des fibres denses ;

- Désorganisation de la gaine fibreuse ;

- Disparition des microtubules du centriole et de l'axonème.

\section{4) INTERPRÉTATION D'UNE NÉCRO- ZOOSPERMIE}

La découverte d'une nécrozoospermie dans un bilan d'infertilité pose souvent des problèmes d'interprétation, tant les étiologies sont difficiles à cerner.

Dans une étude rétrospective portant sur 5049 spermogrammes, Nduwayo et coll. [22] ont retrouvé une nécrozoospermie avec moins de $50 \%$ de formes vivantes dans $4 \%$ des cas. L'étude concomitante des autres paramètres spermiologiques et séminaux, ainsi que l'approfondissement du bilan masculin leur a permis d'identifier 7 catégories de causes qui, en fait, correspondent plutôt à des cadres nosologiques qu'à des étiologies précises. Un contexte infectieux était retrouvé le plus fréquemment (39\% des cas); les autres catégories comprenaient les anomalies du liquide séminal (problème de viscosité, de recueil, de marqueurs prostato-vésiculaires, d'abstinence prolongée...), les anomalies épididymaires (perturbation des marqueurs épididymaires), les pathologies testiculaires (anomalies cliniques, FSH élevée ...), la présence d'auto-anticorps anti-spermatozoïdes (cytotoxiques) et l'existence d'une hyperthermie générale ou locale (varicocèle, exposition professionnelle ...). Dans $20 \%$ des cas, aucune piste étiologique n'a pu être décelée.

La notion d'évolution de la nécrospermie dans le temps semble également très importante. Ainsi, en cas de nécrozoospermie occasionnelle, il est très difficile d'identifier une cause possible et, parmi les étiologies retrouvées, aucu- ne n'apparaît prépondérante. En revanche, si la nécrozoospermie est retrouvée sur plusieurs spermogrammes et, a fortiori, si elle est permanente, il est toujours possible de retrouver un contexte étiologique et, parmi les causes identifiées, les pathologies infectieuses sont largement prépondérantes [22].

La signification d'une nécrozoospermie en termes de pronostic de l'infertilité n'a pas fait, à notre connaissance l'objet d'études publiées à ce jour. A défaut d'étiologies ou de significations précises, il convient d'attribuer à la nécrozoospermie une valeur d'orientation, incitant le médecin à approfondir le bilan andrologique dans plusieurs directions en mettant l'accent sur la recherche de stigmates d'une infection urogénitale. Etant donné que le diagnostic des infections génitales chroniques est particulièrement difficile à poser, en raison du caractère le plus souvent asymptomatique et occulte de ces dernières, il est nécessaire d'inclure dans cette recherche des données cliniques (interrogatoire, examen andrologique ...), paracliniques (échographie prostato-vésiculaire ...) et biologiques (leucospermie, marqueurs séminaux, élastase et autres marqueurs inflammatoires, examen cyto-bactériologique des urines, spermoculture, prélèvements après massage prostatique ...). Les autres orientations du bilan correspondent aux différents contextes étiologiques précédemment cités et sont résumées dans la figure 2 .

\section{CHOIX DU SPERMATOZOÏDE À MICRO-INJECTER}

La technique d'ICSI a révolutionné la prise en charge des stérilités masculines et permet d'obtenir d'excellents résultats, même en cas d'altérations extrêmement sévères du sperme. En effet, il a été démontré que ni la numération, ni la mobilité, ni la morphologie des spermatozoïdes n'influent sur les résultats de l'ICSI. Parmi les paramètres spermiologiques classiques, seule l'absence totale de spermatozoïde mobile après préparation du sperme constitue un facteur de mauvais pronostic en ICSI $[16,21]$, ce qui s'explique par la forte probabilité de micro-injecter dans ces cas un spermatozoïde mort. 


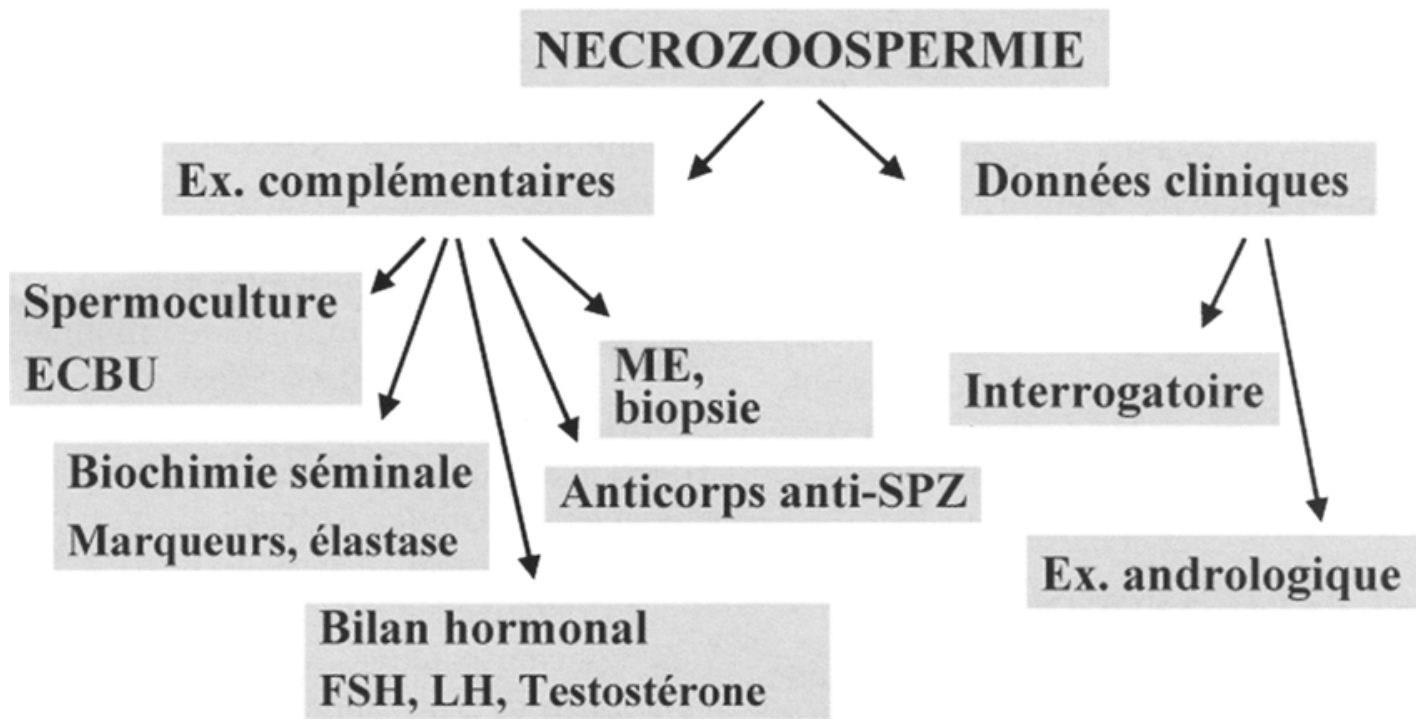

Figure 2: Orientation du bilan masculin en cas de nécrozoospermie (modifié, d'après [22]).

Or mis à part les rares cas de nécrozoospermie totale, il persiste le plus souvent parmi les spermatozoïdes immobiles un certain pourcentage de vivants, susceptibles d'être injectés avec succès. Dans le but de sélectionner ces derniers, les techniques de coloration ne sont pas adaptées, alors que les techniques qui préservent les fonctions du spermatozoïde, comme le HOST et ses dérivés, peuvent y trouver une indication. Une telle sélection s'avère nécessaire en cas d'asthénozoospermie totale et dans certains cas d'ICSI avec spermatozoïdes testiculaires ou cryoconservés.

\section{LE HOST PERMET-IL UNE BONNE SÉLECTION ?}

Pour répondre à cette question, il faut distinguer deux situations bien différentes, suivant qu'il s'agit de sperme frais ou de sperme cryoconservé.

En cas de sperme frais, la comparaison de différentes techniques d'appréciation de la vitalité (HOST, éosine, colorants Hœchst) montre une très bonne corrélation des résultats obtenus [9]. En termes de vitalité, le HOST semble donner les mêmes informations que l'éosine
[28]. En revanche, la corrélation qui existe entre la mobilité et les résultats du HOST n'est pas retrouvée avec l'éosine [15].

Sur le plan clinique, la comparaison des résultats obtenus après ICSI avec des spermatozoïdes immobiles sélectionnés par HOST ou choisis au hasard [6] confirme l'intérêt de cette technique de sélection (taux de fertilisation $43 \%$ versus $26 \%$; taux de clivage $39 \%$ versus $23 \%)$.

En cas de sperme cryoconservé, au contraire, les résultats du HOST ne sont plus corrélés avec les autres techniques, même après correction du pourcentage du HOST, puisque après décongélation, il n'est pas rare d'observer une incurvation flagellaire spontanée dans 15 à $20 \%$ des spermatozoïdes [9].

Dans une étude prospective, utilisant du sperme normo-, asthéno- ou oligozoospermique, Lin et coll. [15] ont montré qu'après décongélation, $33 \%$ des spermatozoïdes étaient jugés vivants d'après le HOST, alors que seuls $9 \%$ l'étaient d'après le test à l'éosine. Inversement, sur les $67 \%$ des spermatozoïdes étiquetés non vivants par le HOST, 7\% restaient capables d'exclure 
l'éosine au niveau de leur tête. Ces discordances pourraient s'expliquer par le fait que les altérations membranaires dues au processus de congélation-décongélation touchent de manière indépendante la membrane de l'acrosome, celle de la tête ou celle du flagelle [20].

L'intégrité membranaire du flagelle n'est donc pas synonyme d'intégrité membranaire de la tête du spermatozoïde. Or il semble que ce soit cette dernière qui importe le plus dans la survie du spermatozoïde après décongélation [7]. De fait, la plupart des auteurs concluent que le HOST classique n'est pas le meilleur outil pour le choix d'un spermatozoïde immobile, cryoconservé, en vue d'une ICSI.

\section{LE HOST EST-IL ANODIN ?}

Devant les taux de fertilisation relativement modestes obtenus avec l'utilisation de spermatozoïdes sélectionnés par le HOST, la question de l'innocuité de ce test sur la survie-même des spermatozoïdes sélectionnés a été soulevée. Parallèlement, des variantes du HOST ont été décrites, parmi lesquelles le test à l'eau, utilisé dans de nombreuses études, en raison de sa simplicité. Introduit par Lomeo [18], il consiste à mélanger $10 \mu \mathrm{l}$ de sperme et $40 \mu \mathrm{l}$ d'eau distillée pour observer des modification osmorégulatrices du flagelle après 5 minutes d'incubation à $37^{\circ} \mathrm{C}$. D'autres milieux hypo-osmolaires peuvent être utilisés, comme la solution de $\mathrm{NaCl}$ à 50 ou à $150 \mathrm{mOsm} / \mathrm{kg}$ [27] ou un milieu de Earle dilué à l'eau à $100 \mathrm{mOsm} / \mathrm{kg}$ [1].

Tsai et coll. [27] ont comparé les effets de différents milieux hypo-osmolaires sur la survie (évaluée par le test à l'éosine) de spermatozoïdes mobiles ou immobiles au départ. Les résultats de cette étude sont résumés dans le
Tableau 1. Il en découle qu'après un temps de contact de seulement une minute, la plupart des spermatozoïdes exposés à l'eau distillée sont morts. Avec la solution hypo-osmolaire recommandée par l'OMS et les solutions de $\mathrm{NaCl}$ à 50 et $150 \mathrm{mOsm} / \mathrm{kg}$, la survie est globalement bonne et légèrement en faveur de la solution de $\mathrm{NaCl}$ à $150 \mathrm{mOsm} / \mathrm{kg}$, en ce qui concerne les spermatozoïdes immobiles. Cette différence devient par contre très significative, même avec les spermatozoïdes mobiles, après 30 minutes d'incubation (c'est-à-dire le temps minimal d'incubation recommandé pour le HOST classique), puisqu'à ce moment-là, $89 \%$ des spermatozoïdes exposés au $\mathrm{NaCl}$ sont toujours vivants, contre seulement $51 \%$ de vivants dans la solution OMS. Le temps d'incubation des spermatozoïdes avec la solution hypoosmolaire devrait donc être le plus court possible.

En étudiant l'évolution de la réponse membranaire à l'HOST en fonction du temps d'incubation Hossain et coll. [13] ont montré que les différents types de gonflement-incurvation flagellaire répondent à une cinétique bien définie. Ainsi, la réponse initiale apparaît à partir de 25 secondes d'incubation, toujours selon le type b (Figure 1), caractérisé par un discret gonflement à l'extrémité du flagelle. Le nombre de spermatozoïdes présentant ce type de réponse augmente rapidement, atteint un plateau vers 3-4 minutes d'incubation, puis diminue progressivement, tandis que d'autres types de réponse commencent à apparaître. Au terme de 30 minutes d'incubation, le type g, caractérisé par un gonflement global du flagelle, devient prédominant. Il semble que les spermatozoïdes, à partir d'un stade initial (le type

Tableau 1: Vitalité (évaluée à l'éosine) de spermatozoüdes mobiles et immobiles après exposition à différentes solutions hypo-osmolaires pendant différentes durées (modifié d'après [27]).

\begin{tabular}{lccc}
\hline $\begin{array}{l}\text { SOLUTION } \\
\text { HYPO-OSMOLAIRE }\end{array}$ & $\begin{array}{c}\text { VitalitÉ APRÈs 1 MINUTEAPRÈs } \\
\text { SPERMATOZOÏDES } \\
\text { MOBILES }\end{array}$ & $\begin{array}{c}\text { SPERMATOZOÏDES } \\
\text { IMMOBILES }\end{array}$ & $\begin{array}{c}\text { 30 MiNUTES } \\
\text { SPERMATOzoÏDES } \\
\text { MOBILES }\end{array}$ \\
\hline Eau distillée & $2 \%$ & $15 \%$ & \\
$\mathrm{NaCl} 50 \mathrm{mOsm} / \mathrm{kg}$ & $82 \%$ & $84 \%$ & $89 \%$ \\
$\mathrm{NaCl} 150 \mathrm{mOsm} / \mathrm{kg}$ & $98 \%$ & $96 \%$ & $51 \%$ \\
Solution OMS & $95 \%$ & $86 \%$ & \\
\hline
\end{tabular}


b), évoluent vers un stade terminal (le type g), soit directement, soit en passant par un ou plusieurs stades intermédiaires (les types $c, d$, e et f). Il est possible que des différences dans la cinétique de la réponse au stimulus hypoosmolaire traduisent des différences de qualité du cytosquelette des spermatozoïdes. De telles différences pourraient avoir un intérêt dans le choix du spermatozoïde à micro-injecter, mais elles échapperont complètement à l'observateur, si le protocole classique est appliqué.

\section{ADAPTATIONS DU HOST}

La technique d'exposition courte au milieu hypo-osmolaire a été adaptée à l'analyse de spermatozoïdes en nombre très réduit, en utilisant une procédure en microgouttes sous huile. Dans une boîte de culture destinée à l'ICSI, le sperme préparé est déposé dans une première microgoutte. Deux autres microgouttes sont disposées à proximité, l'une contenant la solution hypo-osmolaire, l'autre une solution de polyvinylpyrrolidone (PVP) dans du milieu de culture. Cette approche permet de tester individuellement chaque spermatozoïde, en le plaçant avec l'aiguille de micro-injection dans le milieu hypo-osmolaire. Dès qu'une réaction membranaire est visible au microscope (en général en moins d'une minute), le spermatozoïde est repris, placé dans le PVP en vue de l'écrasement du flagelle, puis micro-injecté immédiatement [17].

Parmi 3 couples présentant une immobilité spermatique totale, ce traitement a permis d'obtenir un taux de fertilisation de $76 \%$ et une grossèsse [17]. Une technique similaire a été utilisée avec des spermatozoïdes décongelés [1], mais les résultats cliniques ne sont pas encore publiés.

Une technique alternative de sélection des spermatozoïdes à micro-injecter repose sur le fait que les spermatozoïdes fragilisés décondensent leur noyau dans une solution d'héparine et de glutathion. Après l'incubation dans ce milieu, il suffit de récupérer les spermatozoïdes non décondensés en vue de leur microinjection [29]. Linnocuité et l'efficacité de cette méthode restent cependant à démontrer avant une application à l'espèce humaine.

\section{SPERMATOZOÏDES ET APOPTOSE}

La mise en œuvre des techniques d'ICSI avec des spermes de plus en plus déficients a conduit également à une interrogation sur l'intégrité du génome des spermatozoïdes injectés. En effet, une fragmentation du génome caractéristique de l'apoptose est retrouvée dans les spermatozoïdes, surtout en cas d'altérations spermiologiques importantes.

Une meilleure connaissance des phénomènes apoptotiques dans les spermatozoïdes est nécessaire, afin de pouvoir sélectionner pour l'ICSI des spermatozoïdes qui soient non seulement vivants, mais également exempts de fragmentation de leur ADN et d'autres anomalies qui précèdent la mort programmée de la cellule.

\section{L'APOPTOSE}

L'apoptose est un processus actif, génétiquement déterminé, qui comprend une succession d'altérations morphologiques et fonctionnelles aboutissant à la mort de la cellule. Sur le plan morphologique, sont constatés essentiellement un rétrécissement cellulaire ainsi qu'une condensation et une fragmentation de la chromatine.

Les anomalies fonctionnelles sous-jacentes comprennent surtout une activité endonucléasique qui dégrade l'ADN en fragments d'environ 200 paires de bases, mais également des modifications membranaires avec en particulier une extériorisation des phosphatidylsérines qui passent du feuillet interne de la bicouche phospholipidique au feuillet externe. Enfin, il existe de nombreuses modifications du métabolisme des mitochondries, comme la perte du potentiel de membrane et surtout l'activation de facteurs pro- et anti-apoptotiques, ce qui explique que la mitochondrie est une véritable plaque tournante de l'apoptose.

\section{MOYENS D'ÉTUDE}

De nombreuses techniques permettent d'étudier l'apoptose dans les spermatozoides. La présence de fragments d'ADN double brin peut être détectée par la technique TUNEL (terminal deoxynucleotidyl-transferase-mediated 
dUTP nick end labeling) où, grâce à une enzyme spécifique, des nucléotides marqués (par la biotine ou par un fluorochrome) sont incorporés à l'extrémité $3^{\prime} \mathrm{OH}$ des fragments d'ADN [4, $8,10,23]$.

Les fragments d'ADN double et simple brin peuvent également être mis en évidence par une technique d'électrophorèse alcaline en microgel (comet assay) qui montre une migration en forme de comète, dont la tête correspond au molécules d'ADN intactes et la queue aux fragments apoptotiques [8].

La présence de phosphatidylsérine sur le feuillet externe de la membrane est détectable grâce à la fixation d'annexine $\mathrm{V}$, une protéine qui reconnaît spécifiquement ces phospholipides. Il s'agit d'un marqueur très précoce de l'apoptose [4, 23].

La perte du potentiel de membrane mitochondrial est également un phénomène précoce de l'apoptose et peut être visualisé à l'aide de marqueurs fluorescents qui normalement pénètrent dans la mitochondrie où ils se polymérisent en donnant une fluorescence rouge. En cas de potentiel membranaire mitochondrial abaissé, ces substances restent dans le compartiment cytoplasmique sous forme de monomères qui émettent une fluorescence verte [8].

La fixation d'anticorps monoclonaux anti-ADN simple brin traduit une baisse de la stabilité de I'ADN face à une dénaturation par la chaleur. C'est un marqueur sensible et spécifique des premiers stades de l'apoptose [4].

Enfin, l'étude ultrastructurale en microscopie électronique vient confirmer la fragmentation de la chromatine et la présence d'altérations moins spécifiques, comme l'élargissement des membranes, les redondances membranaires et la présence de vacuoles dans le noyau et le cytoplasme [10].

\section{L'APOPTOSE DANS LES SPERMATO- ZOÏDES}

Des phénomènes apoptotiques peuvent être mis en évidence dans les spermatozoïdes par l'ensemble des techniques précitées. Parmi les déterminismes de ces phénomènes, l'action des radicaux libres, qui proviennent essentielle- ment des spermatozoïdes non fonctionnels et des leucocytes, semble prendre une place prédominante. En effet, ces radicaux libres sont largement en cause dans la survenue de fragmentations de l'ADN [2].

Certains récepteurs membranaires, comme le récepteur Fas, peuvent, après liaison de leur ligand, déclencher la cascade biochimique de l'apoptose. Or il a été démontré que chez les sujets infertiles, en particulier les oligozoospermes, l'expression de ce récepteur à la surface des spermatozoïdes se trouve augmentée [24]. Le fait que ces spermatozoïdes, destinés à la mort cellulaire, arrivent néanmoins à maturité et se retrouvent dans l'éjaculat a conduit à la notion d'apoptose abortive.

Il est également possible qu'au cours de la spermiogenèse, des erreurs d'activité d'une endonucléase soient à l'origine de la mise en route du processus apoptotique. Cette enzyme intervient physiologiquement dans la création et la réparation de points de cassure sur l'ADN, dans le cadre du remplacement des histones par les protamines [8].

Les résultats actuellement disponibles sur l'apoptose des spermatozoïdes montrent que le pourcentage de fragmentation de l'ADN est plus élevé:

- Chez les hommes infertiles [19];

- Chez les hommes porteurs d'une oligo-asthéno-tératozoospermie [19];

- Dans le sperme frais par rapport au spermé préparé [10];

- En présence d'un fort taux de radicaux libres dans le sperme [4].

Le pourcentage de fragmentation de l'ADN est inversement corrélé avec:

- La numération et la mobilité des spermatozoïdes [10];

- Le pourcentage de formes typiques, avec une prédominance des formes microcéphales parmi les spermatozoïdes apoptotiques [10];

- Les taux de fertilisation et de succès de la FIV [25] et de l'ICSI [19].

Il apparaît donc que les phénomènes apoptotiques sont en relation très étroite avec les 
altérations spermiologiques et un mauvais pronostic des techniques d'AMP. Seul le marquage à l'annexine $\mathrm{V}$ a donné des résultats contradictoires, puisque ce marquage a été retrouvé aussi bien dans des préparations de sperme pauvre que dans celles de sperme normal [4]. Il n'est pas encore clairement établi si ce marquage traduit des phénomènes apoptotiques débutants dans ces spermatozoïdes ou s'il résulte directement de la technique de préparation $\mathrm{du}$ sperme, voire de la capacitation des spermatozoïdes.

\section{CONCLUSION}

L'exploration de la vitalité des spermatozoïdes fait partie du bilan spermiologique de base, grâce, notamment, au test à l'éosine-nigrosine. Ce test simple et peu onéreux permet de faire le diagnostic d'une nécrozoospermie et d'orienter alors la poursuite des examens andrologiques de l'homme infertile.

La vitalité est également un élément-clé du choix des spermatozoïdes à utiliser dans le cadre de l'ICSI. Les tests en milieu hypo-osmolaire prennent ici toute leur importance. D'après les connaissances actuelles, il semble préférable de tester individuellement les spermatozoïdes, afin de réduire le plus possible leur temps d'exposition à la solution hypoosmolaire.

La vitalité peut enfin se définir également comme l'absence de tout signe d'initialisation de la mort cellulaire programmée. Depuis quelques années, l'apoptose des spermatozoïdes fait l'objet de nombreuses études qui aboutiront probablement à de nouveaux tests de sélection des spermatozoïdes utilisables en AMP.

\section{REFERENCES}

1. AHMADI A., NG S.C. : The single sperm curling test, a modified hypo-osmotic swelling test, as a potential technique for the selection of viable sperm for intracytoplasmic sperm injection. Fertil. Steril., 1997, 68 : 346-350.

2. AITKEN R.J., GORDON E., HARKISS D. et al. : Relative impact of oxidative stress on the functional competence and genomic integrity of human spermatozoa. Biol. Reprod., 1998, 59 : 1037-1046.
3. BACHTELL N.E., CONAGHAN J., TUREK P.J. : The relative viability of human spermatozoa from the vas deferens, epididymis and testis before and after cryopreservation. Hum. Reprod., 1999, 14 : 3048-3051.

4. BARROSO G., MORSHEDI M., OEHNINGER S. : Analysis of DNA fragmentation, plasma membrane translocation of phosphatidylserine and oxidative stress in human spermatozoa. Hum. Reprod., 2000, 15 : 1338-1344.

5. BLOM E. : A one-minute live-dead sperm stain by means of eosin-nigrosin. Fertil. Steril., 1950, $1: 176$.

6. CASPER R.F., MERIANO J.S., JARVI K.A., COWAN L., LUCATO M.L. : The hypo-osmotic swelling test for selection of viable sperm for intracytoplasmic sperm injection in men with complete asthenozoospermia. Fertil. Steril., 1996, 65 : 972-976.

7. CHAN P.J., TREDWAY D.R., PANG S.C., CORSELLI J., SU B.C. : Assessment of sperm for cryopreservation using the hyposmotic viability test. Fertil. Steril., $1992,58: 841-844$.

8. DONNELLY E.T., O'CONNELL M., McCLURE N., LEWIS S.E.M. : Differences in nuclear DNA fragmentation and mitochondrial integrity of semen and prepared human spermatozoa. Hum. Reprod., 2000, 15 : $1552-1561$.

9. ESTEVES S.C., SHARMA R.K., THOMAS A.J., AGARWAL A. : Suitability of the hypo-osmotic swelling test for assessing the viability of cryopreserved sperm. Fertil. Steril., 1996, 66 : 798-804.

10. GANDINI L., LOMBARDO F., PAOLI D. et al. : Study of apoptotic DNA fragmentation in human spermatozoa. Hum. Reprod., 2000, $15: 830-839$.

11. GARNER D.A., PINKEL D., JOHNSONL.A., PACE M.M. : Assessment of spermatozoal function using dual fluorecscent staining and flow cytometric anaIyses. Biol. Reprod., 1986, 34 : 127-138.

12. GARNER D.L., JOHNSON L.A. : Viability assessment of mammalian sperm using SYBR-14 and propidium iodide. Biol. Reprod., 1995, 53 : 276-284.

13. HOSSAIN A.M., RIZK B., BARIK S., HUFF C., THORNEYCROFT I.H. : Time course of hypo-osmotic swellings of human spermatozoa: evidence of ordered transition between swelling subtypes. Hum. Reprod., $1998,13: 1578-1583$.

14. JEYENDRAN R.S., VAN DER VEN H.H., PEREZPELAES B.G., CRABO B.G., ZANEVELD L.J. : Development of an assay to assess the functional integrity of the human sperm membrane and its relationship to other semen characteristics. J. Reprod. Fertil., $1984,70: 219-228$.

15. LIN M.H., MORSHEDI M., SRISOMBUT C., NASSAR A., OENINGER S. : Plasma membrane integrity of cryopreserved human sperm: an investigation of the results of the hypoosmotic swelling test, the water test, and eosin-Y staining. Fertil. Steril., 1998, 70 : 1148-1155. 
16. LIU J., NAGY JORIS H. et al. : Analysis of 76 total fertilization-failure cycles out of 2732 intracytoplasmic sperm injection cycles. Hum. Reprod., 1995, 10 : 2630-2636.

17. LIU J., TSAI Y.L., KATZ E., COMPTON G., GARCIA J.E., BARAMKI T.A. : High fertilization rate obtained after intracytoplasmic sperm injection with $100 \%$ nonmotile spermatozoa selected by using a simple modified hypo-osmotic swelling test. Fertil. Steril., $1997,68: 373-375$.

18. LOMEO A.M., GIAMBERSIO A.M. : Water-test: a simple method to assess sperm membrane integrity. Int. J. Androl., 1991, $14: 278-282$.

19. LOPES S., SUN J.G., JURISICOVA A., MERIANO J., CASPER R.F. : Sperm deoxyribonucleic acid fragmentation is increased in poor-quality semen samples and correlates with failed fertilization in intracytoplasmic sperm injection. Fertil. Steril., 1998, 69 : 528-532.

20. McLAUGHLIN E.A., FORD W.C.L., HULL M.G.R. : Effects of cryopreservation on the human sperm acrosome and its response to A23187. J. Reprod. Fertil., 1993, $99: 71-76$.

21. NAGY Z., LIU J., JORIS H. et al. : The result of intracytoplasmic sperm injection is not related to any of the three basic sperm parameters. Hum. Reprod., $1995,10: 1123-1129$.

22. NDUWAYO L., BARTHELEMY C., LANSAC J., THARANNE M.J., LECOMTE P. : Conduite à tenir devant une nécrozoospermie. Contracept. Fertil. Sex., $1995,23: 682-685$.

23. OOSTERHUIS G.J., MULDER A.B., KALSBEEKBATENBURG E., LAMBALK C.B., SCHOEMAKER J., VERMES I. : Measuring apoptosis in human spermatozoa: a biological assay for semen quality? Fertil. Steril., 2000, $74: 245-250$.

24. SAKKAS D., MARIETHOS K., St JOHN J.C. : Abnormal sperm parameters in humans are indicative of an abortive apoptotic mechanism linked to the Fas-mediated pathway. Exp. Cell. Res., 1999, 251 : 350-355.

25. SUN J.G., JURISICOVA A., CASPER R.F. : Detection of deoxyribonucleic acid fragmentation in human sperm: correlation with fertilization in vitro. Biol. Reprod., 1997, 56 : 602-607.

26. THOMAS C.A., GARNER D.L. : Post-thaw bovine spermatozoal quality estimated from fresh samples. J. Androl., 1994, 15 : 305-340.

27. TSAI Y.L., LIU J., GARCIA J.E., KATZ E., COMPTON G., BARAMKI T.A. : Establishment of an optimal hypo-osmotic swelling test by examining single spermatozoa in four different hypo-osmotic solutions. Hum. Reprod., 1997, 12 : 1111-1113.

28. VAN DEN SAFFELE J., VERMEULEN L., SCHOONJANS F., COMHAIRE F.H. : Evaluation of the hypoosmotic swelling test in relation with advanced methods of semen analysis. Andrologia, 1992, 24 : 213-217.
29. VENDRELL F.J., RUBIO C., TARIN J.J. : The heparin-glutathion test: an alternative to the hypo-osmotic swelling test to select viable sperm for intracytoplasmic sperm injection. Fertil. Steril., 1998, 70 : 11561161.

30. WHO Laboratory Manual for the Examination of Human Semen and Sperm-Cervical Mucus Interaction. London, Cambridge University Press, 4th edition, 1999.

31. ZAMBONI L. : The ultrastructural pathology of the spermatozoon as a cause of infertility: the role of electron microscopy in the evaluation of semen quality. Fertil. Steril., 1987, $48:$ : 711-734.

\section{ABSTRACT}

The viability of spermatozoa
T. FORGES, P. MONNIER-BARBARINO, B. FOLIGUET

Most of the numerous techniques used to assess sperm viability only have research applications, while only two classical tests, i.e. eosin-Y and hypo-osmotic swelling test (HOST), are currently used in routine sperm analysis to determine the percentage of viable sperm.

A viability rate below $50 \%$ of living sperm defines necrozoospermia, a condition whose clinical significance is fairly difficult to assess as the mechanisms of sperm cell death are still poorly understood. However, even when a precise cause for necrozoospermia cannot be identified, abnormal viability requires further andrological investigations with particular emphasis on clinical and laboratory signs of chronic infection of the male reproductive tract.

Intracytoplasmic sperm injection (ICSI) can yield very good pregnancy rates, even in couples with the most severe forms of male infertility. However, when no motile sperm are available after sperm preparation, the outcome of ICSI is seriously impaired, probably because of a high risk of injecting dead sperm.

In these patients, sperm viability could therefore be assessed by the hypo-osmotic swelling test in order to select only viable 
sperm for ICSI. However, the long incubation time of sperm in the hypo-osmotic solution, as recommended in the classical HOST procedure, has been shown to be detrimental to the spermatozoa. A single sperm test able to assess the viability of each individual spermatozoon within microdroplets covered by mineral oil therefore seems to be preferable. This selection procedure is less suitable in the case of immotile frozen-thawed sperm, as viability does not appear to be reliably predicted by HOST in cryopreserved sperm.

Examination of sperm viability now also evaluates programmed cell death or apoptosis, as apoptotic alterations can be detected in spermatozoa by several techniques. The percentage of apoptotic sperm is correlated with deficient sperm parameters and poor outcome of assisted reproductive techniques. More effective selection procedures are therefore needed in order to identify spermatozoa not only with intact membranes but also with an intact genome to be used for ICSI.

Key words: Viability; Spermatozoa; Eosin-Y; HOST; ICSI; Apoptosis. 